\title{
Multidimensionalidad y validez de la deliberación*
}

Recibido: 23/09/2019 | Revisado: 10/12/2019 | Aceptado: 09/01/2020

DOI: 10.17230/co-herencia.17.32.1

\author{
Julder Gómez** \\ jgomezp5@eafit.edu.co
}

Resumen Muchos problemas deliberativos son multidimensionales: los argumentos a favor y en contra de las soluciones propuestas apelan a valores que pertenecen a distintas dimensiones. Así, por ejemplo, los argumentos a favor de la propuesta de negociar para resolver el problema de un conflicto armado interno pueden apelar a la paz, mientras que los argumentos en contra pueden apelar a la justicia. Esto hace posible que los interlocutores acepten las premisas de un argumento sin comprometerse con su conclusión y que lo justifiquen haciendo énfasis en las premisas del argumento contrario "Sí, una negociación reduciría el número de los muertos por violencia política, pero generaría impunidad, así que no debemos negociar".. Si esta situación fuera insuperable, los argumentos en la deliberación nunca podrían ser válidos, nunca podrían ser tales que la aceptación de las premisas comprometiera a quien las acepta con las conclusiones. En este artículo considero algunas respuestas a este problema: las respuestas de Kock (2009), Govier (2010), Olmos (2016) y la respuesta de Macagno y Walton (2018); señalo algunos problemas que encuentro en estas respuestas e intento resolverlos distinguiendo los casos en que es posible de los casos en los que no es posible superar el problema de la multidimensionalidad en la deliberación.

\section{Palabras clave:}

Deliberación, retórica, argumentos, multidimensionalidad, objetividad, validez, valores.

\section{Multidimensionality and the Validity of Deliberation}

\footnotetext{
Abstract Many deliberation problems are multidimensional: the arguments for and against the proposed so lutions appeal to values that belong to different dimensions. Thus, for instance, the arguments in favor of the proposal to negotiate to solve the problem of a domestic armed conflict may draw upon peace, whereas the arguments against it may invoke justice. This enables spokespeople to accept the premises of an argument without committing themselves to agreeing to its conclusion and justifying such an argument by emphasizing the premises of the opposing argument. "Yes, a negotiation would reduce the number of deaths caused by
}

* Este artículo hace parte de la investigación sobre la teoría integrada de la acción colectiva, financiada por la Universidad EAFIT.

** Profesor del Departamento de Humanidades de la Universidad EAFIT, Medellín-

Colombia. ORCID: 0000-0003-0019-555X 
political violence, but it would also lead to impunity; therefore, we should not negotiate." If this situation were indomitable, the deliberation arguments could never be valid; they could never be such to the extent that the acceptance of the premises would force whoever accepts them to agree to the conclusions. In this paper, some of the answers to this problem are considered: the answers of Kock (2009), Govier (2010), and Olmos (2016) as well as the response of Macagno and Walton (2018). Furthermore, several issues found in these answers are pointed out, and in an attempt to provide a solution, a distinction is made between the cases wherein it is possible and those wherein it is impossible to overcome the problem of deliberation multidimensionality.

\section{Keywords:}

Deliberation, rhetoric, argument, multidimensionality, objectivity, validity, values.

En este artículo considero la cuestión de si, dada la multidimensionalidad de los argumentos en la deliberación, sus conclusiones pueden ser válidas. Las conclusiones de los argumentos suelen considerarse válidas cuando la aceptación de las premisas del argumento compromete a quien las acepta con la aceptación de las conclusiones.

En las discusiones argumentativas de carácter deliberativo, los interlocutores ofrecen y valoran argumentos a favor y en contra de propuestas de cursos de acción, con el ánimo de decidir qué hacer en una situación concreta. Con frecuencia, los argumentos a favor y los argumentos en contra de la misma propuesta pertenecen a distintas dimensiones de un problema. Por ejemplo, en una comunidad en la que la paz y la justicia son dos valores declarados en la misma línea del preámbulo de su Constitución política, los argumentos a favor de una negociación de un conflicto armado pueden atender a la paz mientras los argumentos en contra pueden atender a la justicia. En virtud de esta multidimensionalidad, un interlocutor puede aceptar las premisas de un argumento -si hubiera negociación habría menos muertos por violencia política- y, sin embargo, rechazar su conclusión -aprobemos la negociación de un conflicto armado-, haciendo énfasis en otra dimensión del problema -una negociación dejaría impunes los delitos cometidos durante el conflicto-.

Esta posibilidad, la posibilidad de aceptar las premisas sin aceptar la conclusión, motiva el planteamiento del problema: ¿son, o pueden 
ser válidos los argumentos en la deliberación? Como se verá, esta pregunta se relaciona con otras: ¿se pueden apreciar objetivamente los argumentos?, y iestos se pueden evaluar apropiadamente en términos de verdad? La pregunta principal por la validez de los argumentos prácticos en la deliberación se ha contestado diversamente en el campo de los estudios de la argumentación. En este artículo considero la respuesta de C. Kock (2009), la de T. Govier (2010), la de F. Macagno y D. Walton (2018), y la de P. Olmos (2016). El primero de ellos ha ofrecido una respuesta negativa; la respuesta de Govier (2010), y la de Macagno y Walton (2018) han sido afirmativas; mientras que la propuesta de Olmos (2016) no es una respuesta a la pregunta principal sino a una consecuencia de la respuesta negativa, pero la consideraré porque ayuda a comprender la naturaleza de la discusión. Mi propósito entonces consiste en considerar estas respuestas, señalar algunos problemas que encuentro en ellas, formular unas preguntas que permitan distinguir tipos de discusiones deliberativas, presentar una respuesta afirmativa para dos de esos tipos y una negativa para los tipos restantes.

\section{Una respuesta negativa: la retórica deliberativa de Kock}

A juicio de Kock, las conclusiones de los argumentos en la deliberación no son verdaderas ni falsas porque son propuestas y las propuestas no son verdaderas ni falsas (2017, pp. 26-52). En efecto, enunciados de la forma "Te propongo que hagas $a$ " o "Propongo que hagamos $a$ ", no son inmediatamente verdaderos ni falsos. Sin embargo, una propuesta se puede valorar en términos de verdad o falsedad si se reconstruye como un acto de habla representativo, mediante la proferencia con la que el hablante se compromete con la verdad o falsedad de una proposición. Efectivamente, enunciados de la forma "Es conveniente que hagas $a$ " o "Es conveniente que hagamos $a$ " sí pueden ser evaluados en términos de verdad y falsedad. Kock acepta que esta reconstrucción es posible, pero la estima injustificada en los casos en los que la reconstrucción no puede introducir un modalizador, casos en los cuales el intérprete no tiene una buena razón para afirmar que el hablante quiere que el oyente 
crea que la realización de la acción que le propone es conveniente, debida, obligatoria, etcétera. Y esta restricción es importante porque él entiende que hay casos en los cuales el hablante no quiere que el oyente crea algo para que haga algo más, sino que quiere que el oyente quiera algo para que lo haga (Kock, 2009).

Además, en relación con el asunto principal del presente artículo, Kock sugiere que las conclusiones de los argumentos en la deliberación no son necesarias o válidas en razón del carácter multidimensional de los argumentos que se ofrecen a favor y en contra de una propuesta. La multidimensionalidad de los argumentos consiste en que los argumentos que se ofrecen a favor y en contra de una propuesta suelen pertenecer a distintas dimensiones que no se pueden conmensurar apelando a un común denominador. Puede presentarse, por ejemplo, una situación en la cual se argumente a favor de una propuesta alegando que satisface el valor de la paz en una comunidad y se argumente en contra de la misma arguyendo que no satisface el valor de la justicia; estos argumentos son multidimensionales en cuanto la paz y la justicia sean dos dimensiones distintas no reductibles a una tercera o a un común denominador. En razón del carácter multidimensional de la argumentación en la deliberación, los opositores a una propuesta no necesitan negar los argumentos de quienes están a favor para justificar su oposición; les basta con insistir en las desventajas de la propuesta, no tienen que negar las ventajas de la misma para mantener su renuencia. En este sentido, Kock sugiere que las propuestas no se deducen de sus argumentos: es posible aceptar los argumentos sin aceptar la propuesta, haciendo énfasis en las desventajas.

De acuerdo con esta concepción, optamos por un curso de acción cuando, luego de sopesar sus ventajas con sus desventajas, valoramos más las primeras que las segundas. Kock estima que el peso de cada valor, de cada argumento que apela a una ventaja o a una desventaja, es una propiedad subjetiva relativa a cada miembro de la audiencia. A su entender, la función del debate deliberativo -que es la situación comunicativa en la que parece estar pensandoes ayudar a los miembros de la audiencia a arribar a sus propias conclusiones subjetivas. 
Así, pues, desde el punto de vista de la retórica deliberativa propuesta por Kock, los argumentos empleados en la deliberación carecen de necesidad, carecen de validez, no pueden ser objetivamente considerados ni evaluados en términos de verdad y falsedad.

\section{Respuestas afirmativas}

A diferencia de la retórica deliberativa, otros teóricos de la argumentación contestan afirmativamente, al menos, a algunas de las preguntas referidas: Trudy Govier (2010) ha señalado que una propuesta puede ser valorada en términos de verdad y falsedad; Paula Olmos (2016) ha reconstruido el modo en que, según su interpretación de Aristóteles, las discusiones deliberativas pueden proseguir, más allá de los juicios subjetivos de valor; por último, Macagno y Walton (2018) han sugerido una aproximación modular a la argumentación práctica en la que los argumentos que apelan a valores se justifican mediante argumentos de clasificación, con lo cual se superaría la multidimensionalidad de la argumentación deliberativa.

2.1. T. Govier (2010), en oposición a la distinción entre argumentación teórica y deliberativa propuesta por Kock (2009), ha planteado que la misma se asienta en una falsa dicotomía entre hechos y valores. Arguye que la dicotomía es falsa, siguiendo en esto a H. Putnam (2004): (1) porque los hechos y los valores se imbrican en la selección de las mejores explicaciones, y (2) porque el intento de justificarla apelando a la distinción entre las direcciones de ajuste de los actos de habla fracasa. Esta justificación falla porque (2.1) la distinción entre direcciones de ajuste no es exhaustiva, (2.2) ni es excluyente. No es exhaustiva (2.1) porque hay actos de habla, como las exclamaciones, que no tienen dirección de ajuste. No es excluyente (2.2) porque hay actos de habla, como "Juan es cruel", que, así lo entiende Govier (2010), tienen tanto dirección de ajuste ascendente cuanto descendente, describen y valoran, dándole al agente razones para actuar; lo que ocurre de forma característica con los llamados conceptos éticos densos, de los que "cruel" es un ejemplo. Si estas objeciones son aceptables, entonces, pretendidamente, los argumentos empleados en la deliberación pueden ser evaluados 
en términos de verdad y falsedad, aunque sus conclusiones sean propuestas y no actos de habla representativos.

2.2. Paula Olmos (2016), por su parte, ha expuesto una interpretación de la Retórica de Aristóteles (1363b 5; 1364a 31), de acuerdo con la cual, para los casos en que hay discrepancias sobre cuál de dos cosas es mejor, Aristóteles ofrece una lista de criterios que le permiten al orador compararlas u ordenarlas y jerarquizarlas (1363b 5; 1365b 19). Estos criterios, sin embargo, no son tales que de su consideración se siga con necesidad una única jerarquización posible. De hecho, señala Olmos (2016), es interesante que la mayoría de los criterios presentados por Aristóteles estén acompañados de criterios opuestos que se podrían aducir en favor de una pretensión diferente. Por ejemplo, la escasez puede invocarse para justificar una propuesta de conquistar Persia porque allí hay oro, que es escaso, y la posesión de algo escaso es valiosa; mientras que la abundancia puede emplearse para justificar una propuesta de comerciar con Hesperia, pues allí abunda el hierro, y los minerales abundantes son extremadamente útiles. Así que los oradores pueden usar estos criterios de maneras diferentes, para justificar propuestas diferentes y avanzar en la discusión, sin que la aparente inconmensurabilidad de los valores invocados detenga la discusión.

2.3. Macagno y Walton (2018), finalmente, con el propósito de superar el problema que la multidimensionalidad representa para la descripción y evaluación de los argumentos en la deliberación, proponen enfocarse en la clasificación de los estados de cosas. A su entender, la evaluación de un estado de cosas solo se puede realizar después de clasificar ese estado de cosas:

A state of affairs can be evaluated only after it has been classified. Depending on the way the agent chooses to classify it, the evaluation will change, as it will instantiate a different value. For this reason, classification is a deeper level of reasoning presupposed by practical reasoning (Macagno \& Walton, 2018, p. 536).

Para ilustrar su propuesta, Macagno y Walton reconstruyen y analizan un segmento de un discurso de B. Obama y otro de V. Putin. En el nivel de la reconstrucción que nos interesa, el de la justificación de los juicios de valor, se encuentran las siguientes reconstrucciones: 


\section{Figura 1}

\section{Reconstrucción modular del argumento práctico de Putin}

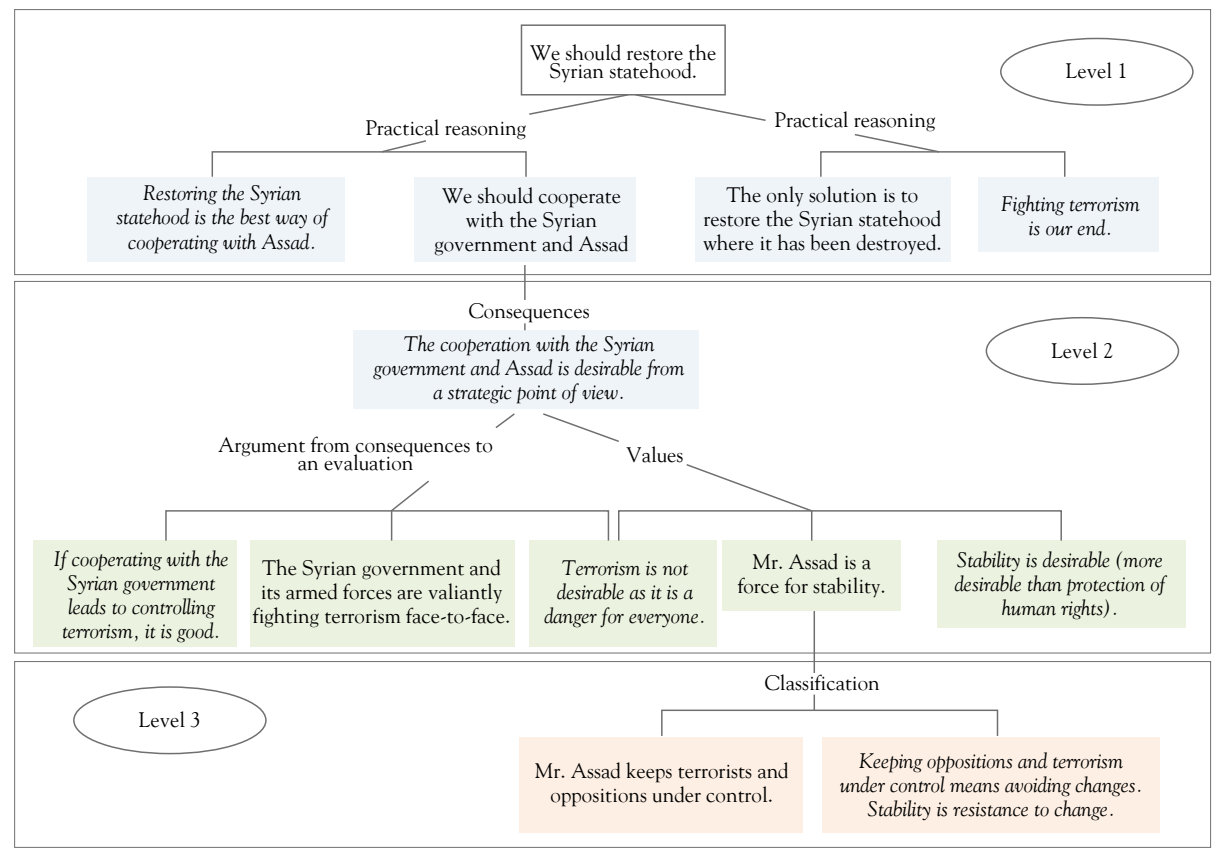

Fuente: F. Macagno \& D. Walton, 2018, "Practical Reasoning Arguments: A Modular Approach", Argumentation, 32(4), p. 541. 
Figura 2

Una reconstrucción modular de la respuesta de Obama al argumento de Putin

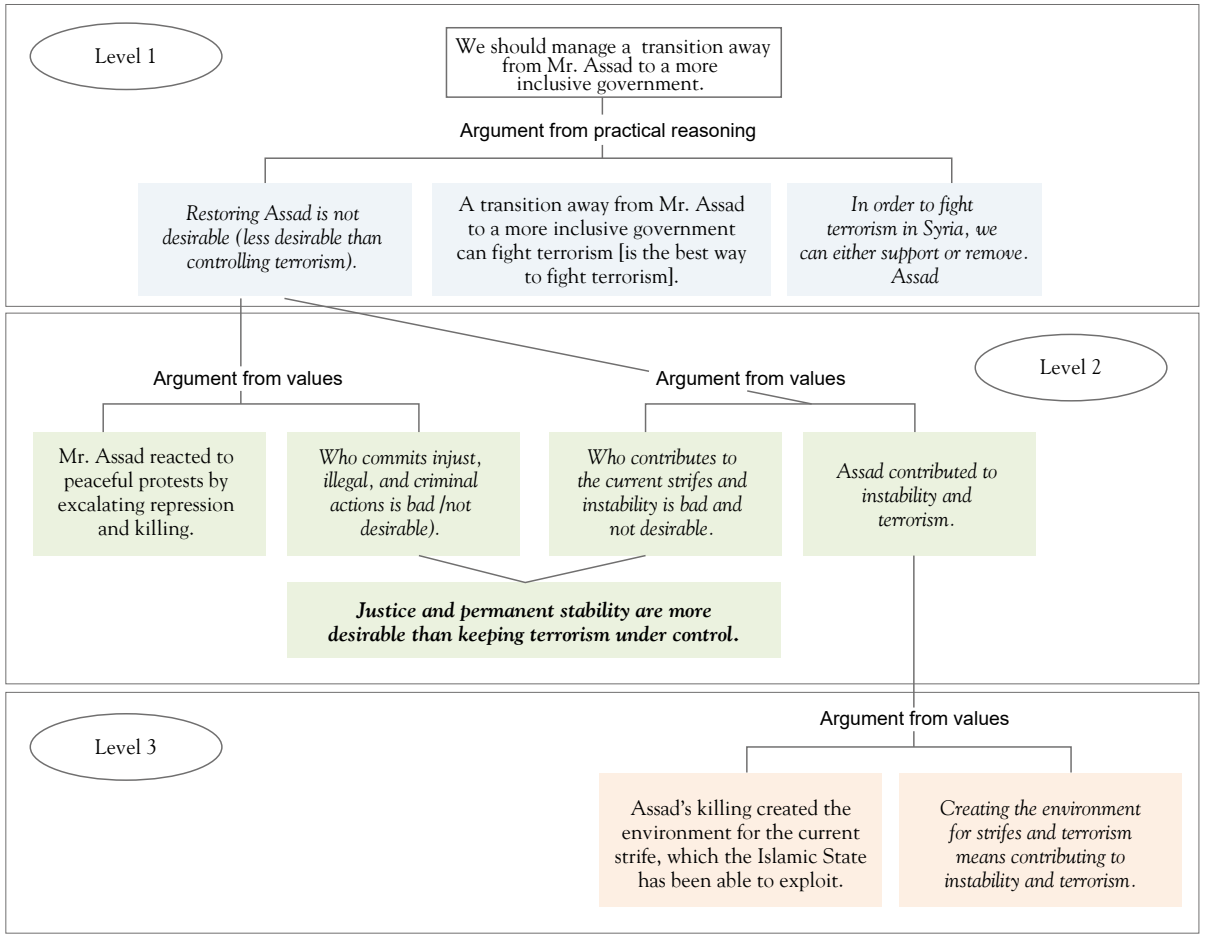

Fuente: F. Macagno \& D. Walton, 2018, "Practical Reasoning Arguments: A Modular Approach", Argumentation, 32(4), p. 542.

Según esta propuesta, la discusión acerca de juicios de valor puede conducirse hacia una discusión acerca de juicios de hecho y, con ello, los análisis de los argumentos en la deliberación se asemejan a los análisis de los argumentos en general: se tornan objetivamente considerables como válidos o inválidos.

\section{Problemas de ambas posturas}

En ambas posturas hay, sin duda, importantes contribuciones a la comprensión de los argumentos en la deliberación y, particularmente, a la elaboración de una respuesta satisfactoria a las preguntas que en 
este texto me interesan. Para ganar en brevedad, en este numeral me detendré únicamente en los aspectos que parece conveniente cuestionar a fin de desarrollar una concepción satisfactoria de la validez de los argumentos empleados en la deliberación.

\subsection{En primer lugar, con respecto a la retórica deliberativa:}

3.1.1. Kock (2009) sugiere que las conclusiones de los argumentos prácticos empleados en la deliberación son propuestas y que no siempre se pueden reconstruir como actos de habla representativos, en particular, porque hay ocasiones en las cuales no se pueden introducir términos modales para comprometer al hablante con la idea de que la realización de la acción es conveniente, debida, obligatoria, etcétera, para el oyente. No todas las propuestas pueden reconstruirse de este modo, pero todas las propuestas y decisiones a favor de las cuales se hayan ofrecido argumentos pueden convertirse en actos de habla representativos según los cuales la realización de la acción propuesta o de la decisión tomada satisface las condiciones teleológicas o axiológicas presentadas en el desarrollo de los argumentos. "Cásate conmigo y sé mi amor", "Pruebe los cigarrillos Camel" y "Ordene ya su auto", son actos de habla directivos que no hay por qué convertir en actos de habla representativos. No obstante, "Cásate conmigo porque te amo", "Pruebe los cigarrillos Camel porque su consumo es placentero" y "Ordene ya su auto porque es el más lujoso del mercado", son argumentos tales que el oyente puede pedirle al hablante explicaciones acerca de cómo es que el amor, el placer y el lujo constituyen motivos para casarse, fumar y comprar un auto, respectivamente.

Las respuestas a estas preguntas comprometen al hablante con que la aceptación de la propuesta o la realización de la decisión satisface ciertas condiciones, teleológicas o axiológicas: así, si la primera es "Porque los esposos que aman a sus esposas las acompañan y la compañía es deseable", el hablante se compromete con que "Si te casas conmigo estarás acompañada"; si la segunda es "Porque el placer hace soportable la vida", el hablante se compromete con que "Si fuma cigarrillos Camel soportará más o mejor la vida"; y si la 
tercera es "Porque la gente que tiene productos más lujosos es mejor tratada en nuestra sociedad", el hablante se compromete con que "Si ordena ya su carro será mejor tratado en nuestra sociedad".

3.1.2. En conexión con las dificultades de la reconstrucción, Kock (2009) propone una distinción entre casos en los que el hablante intenta hacer que el oyente crea algo para que el oyente haga algo y casos en los que el hablante intenta hacer que el oyente quiera algo para que lo haga. En estos últimos casos el acto de hacer que el oyente quiera no se podría reconstruir adecuadamente como un acto de habla representativo. Hay que conceder que algunas formas de hacer querer no comprometen al agente con la aceptabilidad de una proposición. Por ejemplo, hacer querer una comida mostrándola o exponiendo a alguien a su olor, no compromete al agente con ninguna proposición. Pero otras formas de hacer querer sí comprometen al agente: hacer querer describiendo, narrando el proceso de elaboración, sugiriendo consecuencias de su consumo, etcétera, sí comprometen al hablante con la aceptabilidad de su descripción, narración, etcétera; y estas proposiciones pueden ser clasificadas como premisas teleológicas de un argumento práctico, en el esquema sugerido por Fairclough y Fairclough (2013), o como argumentos prácticos que van de los fines a los medios, en la propuesta de Macagno y Walton (2018), pues lo deseado es el fin.

3.1.3. Para los intereses de este artículo, la tesis principal de Kock (2009) consiste en que, dada la multidimensionalidad de los argumentos en los debates políticos, las decisiones son subjetivas porque el peso relativo de los valores es subjetivo: en las situaciones en las que para decidir un curso de acción es necesario determinar cuál de dos valores es más importante, cada uno de los miembros de la audiencia del debate puede llevar a cabo una elección distinta, subjetiva. Aquí, sin embargo, vale la pena preguntar ¿qué significa subjetivo? ¿Significa que su ponderación es expresión de rasgos irracionales, que los juicios de ponderación son básicos, compulsivos, argumentativamente injustificables? Y, además ¿esto ocurre siempre y necesariamente, o solo la mayoría de las veces, o solo en algunas ocasiones importantes? Si esto no ocurriera siempre que es necesario establecer una nueva jerarquía de valores, sería esclarecedor señalar por qué ocurre cuando ocurre. Me ocuparé de contestar estas preguntas en la elaboración de mi propuesta. 


\subsection{En segundo lugar, con respecto a las respuestas afirmativas:}

3.2.1. Haciendo énfasis en el carácter no dicotómico de las distinciones entre las direcciones de ajuste de los actos de habla, T. Govier (2010) ha adelantado un argumento en contra de la dicotomía entre juicios de hecho y juicios de valor que, a su entender, subyace a la propuesta de Kock (2009) y, con ello, la ha rechazado. Esta dicotomía ciertamente puede ser criticada con buenas razones, como Govier (2010) nos recuerda que ha hecho H. Putnam (2004). No obstante, conviene diferenciar entre la reconstrucción de las propuestas que fungen de conclusiones en los argumentos prácticos y la reconstrucción de los juicios de valor que fungen de premisas en los mismos. La primera puede realizarse derivando de los argumentos o premisas las condiciones en las que la realización de la propuesta sería satisfactoria; para la segunda no puede hacerse lo mismo y, aunque una valoración puede estar imbricada en una descripción, puede ocurrir que un juicio valorativo no esté imbricado en ninguna descripción. Así que cuando la toma de una decisión exige una ponderación entre dos juicios de valor en los que ninguna descripción está imbricada, si estos juicios de valor corresponden a dimensiones distintas del problema que se quiere resolver, las conclusiones de Kock (2009) se mantienen, a pesar del carácter no dicotómico de las distinciones entre direcciones de ajuste, de una parte, y entre hechos y valores, de otra.

Por lo demás, puede ser útil considerar que los juicios de valor son actos de habla representativos, contrario a lo que parece suponer Govier (2010), y no Kock (2009), quien, al hablar de las dificultades de la reconstrucción, se ocupa de las propuestas que aparecen en las conclusiones, no de los juicios de valor que aparecen en las premisas. Los juicios de valor, en ciertas condiciones, pueden implicar actos de habla directivos, pero ellos mismos son actos de habla representativos. Así, por ejemplo, "Juan es cruel" en conjunción con "Desaprueba a la gente cruel", en ciertas condiciones, puede implicar "Desaprueba a Juan". El juicio de valor -"Juan es cruel"- funge aquí de premisa, es un acto de habla representativo y puede ser valorado en términos de verdad o falsedad; la propuesta -"Desaprueba a Juan"- es 
un acto de habla directivo y funge aquí de conclusión. La cuestión no es si el juicio de valor que funge de premisa y es un acto de habla representativo puede ser verdadero o falso, se supone que puede serlo; la cuestión es si la propuesta que funge de conclusión y es un acto de habla directivo puede ser verdadera o falsa.

3.2.2. Olmos (2016) recupera los criterios destacados por Aristóteles para argumentar a favor de que algo es mejor que algo más y, al hacerlo, muestra que las discusiones sobre valores no tienen que detenerse ante su inconmensurabilidad y multidimensionalidad. Debe aceptarse que el uso de estos criterios permite que las discusiones no se estanquen en la multidimensionalidad, permite que continúen; ¿pero pueden concluir? Y ¿pueden concluir con necesidad? Una de las propuestas de Kock (2009) consiste en que no pueden concluir con necesidad y, aunque Olmos (2016) no pretende lo contrario, para nuestros propósitos sí es importante señalar que no es evidente que de los criterios expuestos por la rehabilitación aristotélica de Olmos se siga que sea posible concluir con necesidad un argumento práctico que hace parte de una discusión en la que las ventajas a favor de una propuesta pertenecen a una dimensión y las desventajas a otra.

3.2.3. Macagno y Walton (2018), por último, proponen que el problema de la multidimensionalidad se resuelve apelando a los argumentos de clasificación. En sus ejemplos se puede apreciar cómo Putin justifica la pretensión de que es deseable cooperar con Assad partiendo de la clasificación de sus acciones como acciones que mantienen al terrorismo y a la oposición bajo control; mientras que Obama justifica la pretensión de que no es deseable apoyar a Assad partiendo de una clasificación de sus acciones como asesinatos que crearon el ambiente conflictivo que el Estado Islámico aprovecha. Esto, sin embargo, aunque ayuda a comprender cómo puede continuar la discusión, no permite saber cómo puede concluir. En efecto, mientras que una parte hace énfasis en el control, la otra hace énfasis en el conflicto; mientras que una favorece preguntas acerca de cómo mejorar los mecanismos de control, la otra favorece preguntas acerca de cómo acabar el conflicto; mientras que una parte privilegia la dimensión política atinente a las relaciones de poder del problema, la otra privilegia la dimensión humanitaria del 
mismo. La cuestión es si las relaciones de poder en la zona geográfica son más importantes que las vidas de los involucrados o si estas son más importantes que aquellas; clasificar las acciones de Assad como mantenimiento del control es dar más importancia a las relaciones de poder; clasificarlas como asesinatos que generan un ambiente conflictivo es darle más importancia a la vida de los involucrados. Por ello, aunque los argumentos de clasificación juegan un papel importante en las discusiones ejemplificadas, no pueden cumplir la función de superar la multidimensionalidad.

\section{Problemas multidimensionales, situaciones particulares y validez}

En este numeral expongo la tesis de este artículo: hay dos tipos de deliberación en los cuales se pueden resolver problemas multidimensionales de manera objetiva y llegando a conclusiones válidas. Para ello, en primer lugar, retomo una tradición que de Aristóteles a Toulmin asocia la argumentación práctica con los procedimientos clínicos. Este vínculo interesa porque permite hacer explícita la relación entre argumentación práctica, situación particular y objetividad. En segundo lugar, planteo una serie de preguntas con las cuales distingo entre casos deliberativos en los que se pueden resolver problemas multidimensionales y casos en los que no. Los primeros son de dos tipos. Dedico sendos apartados a cada uno de ellos. Al final de este numeral resumo la propuesta.

4.1. Al comienzo del capítulo 7 de Regreso a la razón (Toulmin, 2003, p. 156), puede leerse:

De Aristóteles en adelante, las disciplinas prácticas recurrían a procedimientos 'clínicos', que operaban mediante estructuras temporales, no universales e inmutables, sino que se atenían a los problemas concretos de individuos o situaciones.

De esta concepción clínica de la argumentación práctica, es importante destacar que los argumentos prácticos están orientados a la elección de una decisión en circunstancias particulares. En esto difiere el foco de atención del teórico del foco de atención del 
práctico: el primero se concentra en los rasgos generales y el segundo en las situaciones particulares (Toulmin, 2003, pp. 169-170). Es propio de la teoría intentar determinar lo común a todos los casos y de la práctica intentar comprender lo particular de cada caso. De ahí que la argumentación clínica sea práctica por antonomasia, pues, como se lee en el pasaje de Aristóteles citado por Toulmin:

los que actúan deben considerar siempre lo que es oportuno, como ocurre en el arte de la medicina y de la navegación (Ética Nicomáquea, 1104a, 5-10).

Al médico, mientras atiende a Calias, no le interesa lo que es bueno para la mayoría de los hombres en general sino lo bueno para Calias en su situación particular:

desde luego, el médico no cura a un hombre, a no ser por accidente, sino a Calias, a Sócrates o a cualquier otro (Aristóteles, Metafísica, 981a, 15-20).

Si el médico supiera que para Calias es bueno lo que es bueno para la mayoría de los hombres entonces ya lo habría comprendido, no tendría por qué argumentar; si se presentan discusiones entre médicos acerca de cuál tratamiento es mejor para un individuo es, precisamente, porque lo bueno no es lo mismo para todos los individuos.

Una segunda característica que quisiera destacar de esta concepción de la argumentación práctica es que en ella la objetividad y la valoración coexisten sin impedimentos. En efecto, que lo bueno sea distinto para diferentes individuos no significa que sea epistemológicamente subjetivo. $\mathrm{Si}$ un tratamiento es o no bueno es algo que ha de juzgarse por las consecuencias, si son o no las deseadas por el individuo sometido a tratamiento. Esto sigue siendo así incluso cuando el individuo es un sujeto, un buen tratamiento para él es el que produce los resultados que él desea. La psicología, por ejemplo, trata problemas ontológicamente subjetivos, pero es epistemológicamente objetiva. Tal y como estoy usando aquí estas expresiones, ontológicamente subjetivas son las cosas que no existirían si el sujeto no existiera; epistemológicamente objetivas, de otro lado, son las cosas para cuya evaluación hay criterios acordados -en este caso, si produce los resultados esperados por el paciente-. 
4.2. ¿Esta coexistencia de valoración y objetividad es relevante para todos los tipos de argumentación práctica? Tal sería el caso si lo dicho sobre lo bueno en el ámbito clínico se pudiera mantener para lo bueno en los ámbitos de la ética y de la política. ¿Puede mantenerse? Sugiero que unas veces sí y otras veces no, depende de cómo se contesten las siguientes preguntas en la caracterización de una deliberación dada:

(a). ¿El agente de la deliberación es individual o grupal?

(b). ¿El agente, individual o grupal, ha establecido previamente que algunas cosas pertenecientes a ciertas dimensiones son más importantes que algunas otras pertenecientes a otras dimensiones?

(c). ¿Es posible revisar los valores de la argumentación?

(d). Cuando el agente es grupal ¿los efectos de la decisión sobre los miembros o subgrupos son semejantes?

La objetividad y la valoración coexisten sin impedimentos en dos casos:

(1) cuando el agente es individual, ha establecido previamente alguna jerarquía de valores, o de cosas pertenecientes a distintas dimensiones, y sus valores son revisables;

(2) cuando el agente es grupal, ha establecido previamente alguna jerarquía de valores, sus valores son revisables y los efectos de la decisión sobre los miembros o subgrupos son semejantes.

En los demás casos no, o, por lo menos, no de manera deliberativa, sino mediante otro tipo de argumentación, por medio de la negociación, o de manera no argumentativa, por ejemplo, con el voto.

\subsubsection{El caso de los agentes individuales.}

El problema de la multidimensionalidad se le puede presentar a un agente individual que se siente compelido a tomar una decisión. 
Por ejemplo, se le puede presentar a un individuo a quien le proponen usar un medio de transporte cómodo pero muy contaminante del medio ambiente. El problema aquí consiste en que los argumentos disponibles a favor y en contra pertenecen a distintas dimensiones: a la dimensión de la comodidad y a la del medio ambiente. Ahora bien, este problema solo se plantea si el individuo no le ha dado previamente más peso a una de estas dimensiones. Por lo mismo, una obvia solución posible es decidir a cuál darle más importancia.

Esta última decisión, acerca de a qué darle más importancia, puede ser la conclusión de un ejercicio argumentativo evaluable objetivamente y en términos de verdad o falsedad. Si ha establecido previamente alguna jerarquía de valores pertenecientes a diferentes dimensiones, un agente puede hacer uso de muchos criterios para establecer otra jerarquía de valores multidimensionales, o mejor, para decidir a cuál dimensión de un problema darle más importancia: puede sopesar las consecuencias de diversos órdenes de importancia según su identidad narrativa para ver cuál orden sería más coherente (Smith, 2010), sobre la solución de problemas ulteriores, sobre la consecución de otros fines, sobre los valores y las jerarquías de valor que ha establecido previamente, etcétera. Es cierto que en la evaluación de los diversos órdenes posibles a partir de estos criterios seguramente se presentan de nuevo argumentos a favor y en contra, pertenecientes a diversas dimensiones: puede darle más importancia a la comodidad que al medio ambiente si le da más importancia a su vida en el presente que a las posibilidades futuras, o puede darle más importancia al medio ambiente si le da más importancia al futuro de los niños que a su propio presente. Y también en este caso, si no lo ha hecho previamente, puede hacerlo. En general, si el individuo ha decidido previamente que algunas cosas, no importa cuáles, pertenecientes a ciertas dimensiones, son más importantes que otras cosas cualesquiera, pertenecientes a otras dimensiones, entonces, a partir de esta jerarquía, el individuo puede justificar argumentativamente un nuevo orden de valores. En esto no difiere la deliberación de otros tipos de argumentación: para argumentar hace falta que previamente se hayan establecido algunas cosas.

Sin embargo, este ejercicio argumentativo no sería posible 
cuando las jerarquías de valor y los valores previamente establecidos no fueran revisables, cuando fueran básicos o compulsivos. Estas dos distinciones, (i) entre juicios compulsivos de valor y juicios no compulsivos de valor, y (ii) entre juicios básicos de valor y juicios no básicos de valor, son retomadas de Sen (1967) por Putnam (2004, pp. 94-98) para la consideración de las razones en la ética.

Los juicios compulsivos de valor implican imperativos absolutamente incondicionales, imperativos que deben cumplirse con independencia de cualquier razón que pudiera haber para no hacerlo. Los juicios básicos de valor, por otro lado, son los que el agente mantendría a pesar de cualquier revisión de supuestos fácticos. Tanto los primeros cuanto los segundos son juicios de valor no revisables. Por ejemplo, los juicios de valor "Es valioso y, por tanto, hay que cuidar el medio ambiente" o "Es valioso y, por tanto, hay que estar cómodo" serían compulsivos para un agente que estuviera dispuesto a obedecerlos en cualquier circunstancia y sin consideración ulterior alguna; al tiempo que serían básicos para un agente que los mantuviera a pesar de cualquier cambio en la información disponible sobre el medio ambiente o la comodidad.

Putnam (2004) retoma de Sen (1967) la anotación, importante para el estudio de la deliberación, de que la mayor parte de los juicios de valor no son compulsivos ni básicos. La mayoría de las veces, quienes deliberan invocan valores como razones prima facie para actuar, en ciertas circunstancias, no en cualquier circunstancia; así mismo, la mayoría de las veces, quienes invocan valores como razones para actuar lo hacen a la luz de la información disponible, no con total independencia de ella. Por ejemplo, la mayoría de quienes invocan la comodidad y el medio ambiente como razones para actuar están dispuestos a hacer una excepción, a estar incómodos o a contaminar, en algunos casos; y la mayoría de ellos dejaría de invocar estas razones si descubriera que la comodidad es altamente dañina para la salud mental o que el medio ambiente ya está irremediablemente perdido. Así que, por lo menos la mayoría de las veces, quienes han establecido o aceptado previamente un valor o una jerarquía de valores multidimensionales pueden hacer revisiones que les permitan establecer nuevos órdenes de importancia entre valores pertenecientes a distintas dimensiones de un problema y 
resolver, así, el escollo que la multidimensionalidad de los valores representa para la deliberación.

En cambio, la multidimensionalidad resultaría argumentativamente irresoluble para un agente confrontado con una propuesta a favor y en contra de la cual se presentaran valores básicos y compulsivos pertenecientes a distintas dimensiones. En nuestro ejemplo, si un agente aceptara los imperativos de estar cómodo y cuidar el medio ambiente sin importar las circunstancias ni la información fáctica disponible, en una situación en la que tuviera que desplazarse y no hubiera más alternativas de transporte que incómodos trenes y autos contaminantes, ese agente no podría resolver argumentativamente el problema de la multidimensionalidad. No obstante, una situación como esta es, por lo menos, excepcional.

4.2.2. El problema de la multidimensionalidad puede planteársele también a un grupo. En lugar de pensar en una situación en la que un individuo tiene que elegir entre transportarse cómodamente y cuidar el medio ambiente, podemos ahora pensar en una situación en la que un grupo tiene que hacer esta misma elección. También en este caso los argumentos a favor y en contra pertenecen a dimensiones diferentes. No obstante, a diferencia de lo que encontramos en el caso individual, cuando se trata de grupos podemos hallar que unos subgrupos o individuos se pronuncian a favor de una propuesta, otros a favor de otra y, quizás, algunos más dudan entre las alternativas. De nuevo, el problema solo se presenta si a la luz de las reglas y valores del grupo (formales, informales, implícitos o explícitos) no es obvio si el medio ambiente es más importante que la comodidad o si, al contrario, esta es más importante que aquel.

En el caso grupal, para resolver el problema de manera argumentativa se necesita algo más que haber establecido previamente alguna jerarquía de valores multidimensionales y algo más que valores revisables: se requiere que las consecuencias de la decisión afecten de manera semejante a los miembros del grupo; se precisa que afecten de manera semejante, entre otros elementos, sus fines, sus reglas, sus capacidades y sus identidades narrativas, tomados ya como individuos o ya como subgrupos.

Los fines y las reglas no son exactamente lo mismo cuando se trata de individuos y cuando se trata de grupos. Un individuo 
puede suscribir fines o reglas incompatibles entre sí. Empero, el reconocimiento de ello en una situación en la que tiene que tomar una decisión que afecta esos fines o esas reglas lo presiona para priorizar unos fines o reglas en detrimento de otros u otras. Esto es posible porque todos esos fines y esas reglas son fines y reglas del mismo individuo. En cambio, no todos los fines y reglas de los miembros de los grupos son fines y reglas de los grupos. Cuando los fines o reglas de un subgrupo entran en conflicto con los de otro no se genera la misma presión para priorizar que se genera en el caso individual porque en este caso grupal priorizar unos fines o reglas equivale a priorizar un subgrupo, lo que resulta inaceptable para el subgrupo desfavorecido. Así, el valor social de la comodidad del transporte se alinea con el fin subgrupal del enriquecimiento de los transportadores de una manera que no se alinea con el fin subgrupal de los padres preocupados por el futuro de sus hijos y, al contrario, el fin social de la protección del medio ambiente se alinea con el fin subgrupal del cuidado del futuro de los hijos de una manera que no se alinea con el fin subgrupal del enriquecimiento de los transportadores. En esta situación cada subgrupo puede apelar a un valor grupal distinto, la comodidad o el medio ambiente, cuya subordinación a otro valor grupal no puede aceptar sin renunciar a sus fines o reglas subgrupales e individuales. Por esta razón, en estas situaciones es difícil concertar una nueva jerarquía de valores por la vía de la argumentación deliberativa y se hace impajaritable o bien dar paso a otro tipo de argumentación, a la negociación, o bien hacer uso de algún dispositivo para la toma de decisiones colectivas en escenarios de disenso, por ejemplo, del voto.

Lo mismo ocurre con las capacidades y la identidad narrativa: cuando las consecuencias de las decisiones grupales no afectan de manera semejante las capacidades y la comprensión narrativa de sí que tienen los subgrupos, el establecimiento de una nueva jerarquía de valores se dificulta porque implica una nueva jerarquía entre los subgrupos integrantes del grupo. En efecto, para continuar con el ejemplo, darle más importancia al medio ambiente que a la comodidad del transporte puede implicar, entre otras cosas, darle más importancia al futuro de los niños que al presente de los transportadores, lo que puede parecerle injusto a los transportadores. 
Una nueva jerarquía como esta afectaría de maneras distintas a los transportadores y a los niños: debilitaría a los primeros en beneficio de los segundos. Asimismo, una nueva jerarquía, en estas condiciones, sería más fácilmente comprensible como conveniente para unos subgrupos que para otros: dado que nuestra concepción narrativa (Lakoff, 2008, pp. 22-36) de los niños implica que han de crecer y no que deben transportarse, mientras que nuestra concepción narrativa de los transportadores implica que se lucran de ofrecer un transporte más cómodo y no que tienen hijos o que transportan niños, es más fácil para el primer subgrupo que para el segundo comprender la conveniencia de esta nueva jerarquía.

En cambio, cuando un grupo comparte fines, reglas, identidad narrativa y cuando, además, los efectos de las decisiones repercuten de maneras semejantes sobre sus capacidades, cuando eso ocurre, ese grupo puede establecer deliberativamente una nueva jerarquía entre las dimensiones a las cuales pertenecen los argumentos a favor y en contra, sin con ello privilegiar un subgrupo en detrimento de otro, por lo cual el problema puede ser abordado por el grupo como "nuestro" problema, y las soluciones como las mejores "para todos". En estos casos una nueva jerarquía puede ser establecida de manera objetiva como la mejor para los intereses del grupo.

Ahora bien, que se pueda establecer objetivamente una nueva jerarquía no implica que se pueda establecer de manera universal, pues lo mejor para ese grupo será lo mejor en sus circunstancias, es decir, dados sus particulares fines, reglas, capacidades y autocomprensión. Es claro que para otro grupo que tuviera otras condiciones y otras razones para actuar, otro debería ser el nuevo orden de valores.

4.2.3. Como queda dicho en los dos numerales anteriores (4.2.1 y 4.2.3), en la deliberación sobre problemas multidimensionales la valoración y la objetividad epistemológica pueden coexistir sin impedimento cuando el agente ha establecido previamente alguna jerarquía entre valores multidimensionales, cuando sus valores son revisables y cuando, en el caso de que el agente sea grupal, las consecuencias de la adopción de una nueva jerarquía multidimensional afectan de manera semejante a los integrantes del grupo.

En estos casos, el agente, individual o grupal, puede averiguar críticamente cuál de dos posibles jerarquías de valores 
multidimensionales sería mejor para él, siendo el agente y sus circunstancias como son. Para esto el agente puede servirse de muchos criterios: puede comparar las consecuencias de adoptar una u otra jerarquía sobre las adoptadas previamente, sobre los fines establecidos con antelación, sobre las capacidades del agente para continuar con su agenda, sobre su identidad narrativa, etcétera. En estos casos las conclusiones de los argumentos prácticos pueden ser válidas, en el sentido en que la aceptación de la nueva jerarquía de valores y de lo que esta implica -una nueva jerarquía de fines y una nueva descripción de la situación- compromete al hablante con la aceptación de una propuesta. En efecto, la nueva jerarquía disminuye la importancia de los argumentos asociados con el valor subordinado y encarece la de los asociados con el valor superior.

La diferencia entre la argumentación práctica en estos casos y la argumentación teórica reside en que la primera está orientada a la toma de decisiones oportunas en situaciones particulares, mientras la segunda está orientada a la comprensión de lo general; reside en la filiación de la primera con lo que en el pasaje citado Toulmin denomina "procedimientos clínicos". En términos argumentativos esto significa que los valores, los fines y las consecuentes descripciones de las circunstancias que aparecen en los argumentos prácticos han de ser siempre y solamente los que el agente, individual o colectivo, que ha de tomar la decisión, declare como suyos. De ello no se sigue que esta clase de argumentación sea arbitraria, pues el agente no tiene que elegir arbitrariamente las premisas, sino que las puede obtener al término de un ejercicio crítico de argumentación; tampoco se sigue de ello que la conclusión sea inválida, ya que una nueva jerarquía de valores puede disminuir la importancia, ora de los pro, ora de los contra de una propuesta, lo cual genera una situación en la que si se aceptan las premisas se contrae un compromiso con la conclusión. Lo que sí se sigue de los considerandos anteriores es que lo que se determina como bueno en las conclusiones de estos argumentos es bueno solo para el agente en cuestión, aun si se pretende universalmente que es bueno solo para él. En este sentido la máxima "Haz que lo bueno para ti sea universalmente bueno para ti" puede ser una apropiada descripción del cometido de una deliberación ya 
individual o ya de un grupo cuyos miembros se ven afectados de manera semejante por las consecuencias de sus decisiones colectivas.

\section{Conclusiones}

5.1. La pregunta principal de este artículo ha sido si, dada la multidimensionalidad de los argumentos en la deliberación, sus conclusiones pueden o no ser consideradas como conclusiones válidas. En el planteamiento de esta pregunta se ha empleado el adjetivo "válidas" para clasificar las conclusiones de los argumentos que se está comprometido a aceptar si se han aceptado las premisas. La cuestión se ha planteado porque cuando los argumentos a favor de una propuesta formulada en una deliberación pertenecen a una dimensión y los argumentos en contra pertenecen a otra, los interlocutores pueden aceptar las premisas de un argumento, a favor o en contra de una propuesta, sin comprometerse con la conclusión, haciendo énfasis en las premisas del argumento contrario.

He sugerido que este problema se le plantea a un agente, individual o grupal, solo si ese agente no ha establecido previamente una jerarquía de valores entre las dimensiones del problema y que, por tanto, el problema se puede resolver si se establece argumentativamente una nueva jerarquía de valores entre estas dimensiones. He sugerido que esto es posible en algunos casos y que, en virtud de la nueva jerarquía de valores, la aceptación de las premisas de un argumento compromete a quien las acepta con la aceptación de la conclusión. En efecto, la nueva jerarquía de valores no niega, pero sí resta valor o importancia a las premisas de los argumentos contrarios. He sugerido que el establecimiento de una nueva jerarquía de valores y, con ello, la ulterior resolución argumentativa del problema, es posible en dos casos:

(1) cuando el agente es individual, ha establecido previamente alguna jerarquía de valores, o de cosas pertenecientes a distintas dimensiones, y sus valores son revisables;

(2) cuando el agente es grupal, ha establecido previamente alguna jerarquía de valores, sus valores son revisables y los 
efectos de la decisión sobre los miembros o subgrupos son semejantes.

La institución de nuevos órdenes de valores puede realizarse mediante la búsqueda de respuestas aceptables para el agente a preguntas por las consecuencias de los diversos órdenes posibles sobre las jerarquías previamente establecidas, los valores ya fijados, los fines deseados, las capacidades del agente, etcétera. Un agente individual puede resolver el problema de la multidimensionalidad encontrando respuestas aceptables a estas preguntas.

En el caso de los agentes grupales el problema se resuelve si las respuestas son consecuencias semejantes para todos y no se resuelve si, a la luz de las respuestas, las consecuencias sobre los miembros o subgrupos son desemejantes. No quiero con esto decir que no se resuelve cuando hay desacuerdos acerca de las respuestas, sino que no se resuelve cuando las decisiones afectan de manera desemejante a los miembros del grupo. En el ejemplo de una comunidad que debe elegir entre transportes cómodos pero contaminantes y transportes no contaminantes pero incómodos, el problema de la multidimensionalidad no se resuelve cuando a la pregunta ¿cómo se ven afectadas mis capacidades con esta medida? los padres de los niños encuentran una respuesta aceptable -me debilitan- y los transportadores encuentran otra respuesta aceptable -me fortalecen-. Las dos respuestas son aceptables, pero a un subgrupo le dan razones a favor de una propuesta y al otro le dan razones en contra. En cambio, cuando las respuestas a las preguntas por las consecuencias de diversos órdenes de valor posibles son semejantes para los subgrupos, la ponderación de estos órdenes puede arrojar una nueva jerarquía de valores a partir de la cual se resuelve el problema de la multidimensionalidad.

5.2. Una de las características llamativas del problema de la multidimensionalidad consiste en que frecuentemente los grupos tienen dificultades resolviendo argumentativamente problemas multidimensionales, mientras que los sujetos individuales los resuelven con mayor facilidad. A primera vista, esto sugiere que dicha solución es subjetiva. No obstante, siguiendo en esto una indicación de Toulmin y, antes de él, de Aristóteles, he expuesto que la clave no es aquí 
la subjetividad, sino la situación particular. Los sujetos individuales resuelven más fácilmente los problemas multidimensionales porque atienden a sus situaciones particulares. También los grupos pueden resolver argumentativamente problemas multidimensionales cuando las situaciones particulares de los subgrupos son afectadas de manera semejante por las consecuencias de las decisiones grupales. Tanto los sujetos individuales cuanto los grupos pueden arribar objetivamente a conclusiones válidas, haciendo de las preguntas críticas pertinentes criterios para la instauración de una nueva jerarquía, con arreglo a la cual se resuelva el problema. Estas cuestiones permiten comparar las consecuencias de la adopción de un nuevo orden de valores sobre los valores y las jerarquías previamente aceptados, sobre los fines fijados con antelación, sobre las descripciones de las situaciones problemáticas, sobre las capacidades del agente, sobre su identidad narrativa, etcétera.

5.3. Cada una de las respuestas a cada una de estas preguntas se puede evaluar en términos de verdad o falsedad. Las consecuencias de la admisión de una nueva jerarquía de valores pueden ser conjeturadas de tal manera que se pueda constatar ulteriormente si esas conjeturas eran correctas o incorrectas. Estas hipótesis se expresan mediante actos de habla representativos que comprometen al hablante con la verdad de una proposición. Es cierto que el acto de habla declarativo mediante el cual se expresa una nueva jerarquía y, en segundo lugar, el acto de habla compromisorio de la decisión de acoger y ejecutar una propuesta a la luz de una nueva jerarquía, estos actos de habla no son, evidentemente, actos de habla representativos. No obstante, es posible derivar de los argumentos a favor de la nueva jerarquía la condición con la cual esta sería satisfactoria, a saber, sería satisfactoria si su establecimiento tuviera las consecuencias conjeturadas; del mismo modo, es posible derivar de los argumentos a favor de la propuesta coherente con la nueva jerarquía las condiciones teleológicas y axiológicas bajo las cuales su ejecución sería satisfactoria, esto es, sería satisfactoria si con su realización se consiguiera el fin y el valor propuestos.

Así, si la razón para darle más importancia al medio ambiente que a la comodidad era afianzar los valores familiares, enfatizar jerarquías previas en las que el futuro es más importante que el 
presente, encarecer una identidad narrativa de la comunidad como una comunidad de familias, y si el fin de la elección de los medios de transporte no contaminantes sobre los medios de transporte cómodos era que los niños tuvieran mejor salud en el futuro, entonces, todo esto habrá sido correcto si, por una parte, con la nueva jerarquía se acentúan esos valores, esas antiguas jerarquías y ese esquema narrativo, y si, por otra parte, con la decisión se consigue que los niños tengan mejor salud en el futuro.

5.4. En el caso de las decisiones de los agentes grupales, resulta indispensable averiguar lo antes posible la respuesta a la pregunta ¿en realidad se ven afectados los subgrupos de manera semejante por las soluciones propuestas? Cuando ese no es el caso la deliberación puede no ser la práctica argumentativa adecuada a la resolución del problema, quizá lo sea la negociación; en cambio, cuando sí es el caso, es razonable exigirle a cada uno de quienes participan en la discusión que exponga sus razones para darle más importancia a una dimensión del problema que a otra, que diga por qué debería el grupo adoptar una nueva jerarquía de valores: ¿por qué debería importar más la justicia que la paz o la paz que la justicia?, ¿por qué debería importar más la comodidad que el medio ambiente o el medio ambiente que la comodidad? Esta puede ser entendida como una obligación dialéctica $\mathbf{I}$

\section{Referencias}

Aristóteles. (1985). Ética Nicomáquea. Ética Eudemia (J. Pallí Bonet, Trad.). Gredos.

Aristóteles. (2008). Metafísica (T. Calvo Martínez, Trad). Madrid: Gredos.

Fairclough, I., \& Fairclough, N. (2013). Political Discourse Analysis: A Method for Advanced Students. Routledge.

Govier, T. (2010). Govier: Reflections of Fact, Values and Argument. En C. Reed \& C. W. Tindale (Eds.), Dialectics, Dialogue and Argumentation (pp. 19-31). College Publications.

Kock, C. (2009). Arguing for Different Types of Speech Acts. OSSA Conference Archive, 90, 1-12. https://bit.ly/3d4Q96J

Kock, C. (2017). Deliberative Rhetoric: Arguing about Doing. Windsor 
Studies in Argumentation. https://doi.org/10.22329/wsia.05.2017

Lakoff. (2008). The Political Mind: Why You Can't Understand 21st-Century American Politics with an 18th-Century Brain. Viking.

Macagno, F., \& Walton, D. (2018). Practical Reasoning Arguments: A Modular Approach. Argumentation, 32(4), 519-547. https://doi. org/10.1007/s10503-018-9450-5

Olmos, P. (2016). Meta-Argumentation in Deliberative Discourse: Rhetoric 1360b05-1365b21. OSSA Conference Archive, 73, 1-18. https://bit. ly/392HcYh

Putnam, H. (2004). El desplome de la dicotomía hecho-valor y otros ensayos. Paidós.

Sen, A. (1967). The Nature and Classes of Prescriptive Judgements. The Philosophical Quarterly, 17(66), 46-62. https://doi.org/10.2307/2218365

Smith, M. N. (2010). Practical Imagination and its Limits. Philosopher's Imprint, 10(3), 1-20. https://bit.ly/2IRXtVl

Toulmin, S. (2003). Regreso a la razón: El debate entre la racionalidad y la experiencia. Península. 